\title{
Magnetic Lines of Force Deformed by Motion of a Conducting Fluid ( I )
}

\author{
Yukio HaGiWARA \\ Earthquake Research Institute, University of Tokyo \\ (Received May 20, 1967)
}

\begin{abstract}
Motion of a conducting fluid distorts magnetic lines of force permeating the fluid. The magnetic field is dragged and amplified by the fluid motion. It has been thought that such an interaction between motions and magnetic fields is actually taking place within the earth's core. Time-dependent behaviour of magnetic lines of force deformed by a pair of convection cells is obtained by solving a non-steady equation of magnetic field in a conducting fluid with the intention of examining the fields above convective up-and downflows in the core's top layer. The computation is successively made for initially uniform as well as non-uniform fields by a method similar to the relaxation technique on the assumption that the fluid velocity is fixed and prescribed.The results obtained here qualitatively indicate that up- and down-flows in the core's top layer may possibly induce the nondipole highs and lows at the earth's surface.
\end{abstract}

\section{Introduction}

Bullard et al. (1950) noticed that there is a marked magnetic non-dipole low in the Central Pacific Ocean. According to the paleomagnetic measurements of rocks sampled on islands in the Central Pacific, the non-dipole low seems likely to have been persistent there for a long period of geological time. We also see that the amplitude of geomagnetic secular variation there is small. One of the possible causes of the non-dipole low might be the distortion of the magnetic field by a convective down-flow of core material. Vestine (1965) argued for the down-flow on the basis of an equation for a perfect conductor, treating the magnetic field without any distinction between the interior and exterior of the core. However, as suggested by the dynamo theory, the very large toroidal field vanishes at the core-mantle boundary, being identically zero in the mantle, while the poloidal field which is much smaller than the toroidal field is produced by an interaction between the toroidal field and convective fluid motions within the core. What we can observe at the earth's surface is the poloidal field. In the author's opinion, proper accounts of the toroidal and poloidal fields should be added to Vestine's theory. Rikitake (1967) treated a similar problem on the basis of a theory advanced by Nagata and Rikitake (1961) in which a plausible physical mechanism for the production of the non-dipole field had been taken into account. Rikitake also estimated the distribution of the horizontal velocity at the core's surface by analyzing the magnetic potential of the non-dipole field observed at the earth's surface.

This author intends in the present paper to estimate the deformation of magnetic lines of force by convecting currents within the earth's core. It is expected that the field weaken- 
ing in the Central Pacific Ocean may be explained by a down-flow associated with a sink. ing of the convecting cells as will be discussed in this paper. The fundamental equation of the non-dipole field, which is the same as the one advanced by Nagata and Rikitake (1961), will be numerically solved on the assumption that patterns of convecting cells are prescribed. In Section 2, a mathematical formula which is conveniently applicable to an actual computation will be derived from the fundamental equation. Actual computation will be made on a HITAC 5020 computer for a model in which the magnetic field is uniform inside the core when the fluid is stationary, and also for a model in which a non-uniform field is assumed. The results of the computation will be given in Section 3 .

\section{Fundamental Equation for the Magnetic Field.}

The fundamental equation that controls a non-steady magnetic field in a conducting fluid is written as

$$
\frac{\partial \overrightarrow{\boldsymbol{H}}}{\partial t}=\frac{1}{4 \pi \sigma} \nabla^{2} \overrightarrow{\boldsymbol{H}}+\operatorname{curl}(\overrightarrow{\boldsymbol{v}} \wedge \overrightarrow{\boldsymbol{H}})
$$

where $\overrightarrow{\boldsymbol{H}}$ is the magnetic field, $\overrightarrow{\boldsymbol{v}}$ the velocity of the fluid and $\sigma$ the electrical conductivity, assuming that the fluid is incompressible. We can introduce generating functions $U$ and $\Psi$ for $\overrightarrow{\boldsymbol{H}}$ and $\overrightarrow{\boldsymbol{v}}$ respectively in the case of a two-dimensional treatment with respect to the $x$ and $z$ coordinates, because $\operatorname{div} \overrightarrow{\boldsymbol{H}}=0$ and $\operatorname{div} \overrightarrow{\boldsymbol{v}}=0$. Then the $x$ and $z$ components of $\overrightarrow{\boldsymbol{H}}$ and $\overrightarrow{\boldsymbol{v}}$ can be expressed as

$$
\overrightarrow{\boldsymbol{H}}=\left(-\frac{\partial U}{\partial z}, \frac{\partial U}{\partial x}\right)
$$

and

$$
\overrightarrow{\boldsymbol{v}}=\left(-\frac{\partial \Psi}{\partial z}, \frac{\partial \Psi}{\partial x}\right)
$$

and by substituting (2) and (3) into (1), we get

$$
\frac{\partial U}{\partial t}=\frac{1}{4 \pi \sigma} \nabla^{2} U-\frac{\partial \Psi}{\partial x} \frac{\partial U}{\partial z}+\frac{\partial \Psi}{\partial z} \frac{\partial U}{\partial x} .
$$

Assuming that the generating function of the magnetic field consists of a term which is independent of $x$ and a term produced by the interaction between the motion and the magnetic field, we put

where

$$
U=-F(z)+u(x, z, t),
$$

$$
F(z)=\int_{0}^{z} H_{0}\left(z^{\prime}\right) d z^{\prime} .
$$

As for typical cells of convection, we adopt a generating function of the fluid velocity given by

$$
\Psi=A \sin \frac{\pi x}{L} \sin \frac{\pi z}{L}
$$


where $L$ indicates the diameter of the convecting cell. By substituting (5), (6) and (7) into (4), we obtain

$$
\frac{\partial u}{\partial t}=\frac{1}{4 \pi \sigma}\left\{\nabla^{2} u-H_{0}^{\prime}(z)\right\}-\frac{\pi A}{L}\left[\left\{\frac{\partial u}{\partial z}-H_{0}(z)\right\} \cos \pi x \sin \pi z-\frac{\partial u}{\partial x} \sin \pi x \cos \pi z\right]
$$

within the earth's core. It is seen that (8) holds good even for the space outside the convection cell provided $A=0$. In order to solve the equation (8), the author makes use of a technique similar to the relaxation method. Taking a time step as $\Delta t, \partial u / \partial t$ can be approximately rewritten as

$$
\frac{\partial u}{\partial t}=\frac{u(x, z, t+\Delta t)-u(x, z, t)}{\Delta t}
$$

and covering the space with a net of square mesh, $\nabla^{2} u$ is given by

$$
\begin{array}{r}
\nabla^{2} u=\frac{1}{s^{2}}\{u(x+s, z, t)+u(x-s, z, t)+u(x, z+s, t) \\
+u(x, z-s, t)-4 u(x, z, t)\},
\end{array}
$$

where $s$ is a spacing between digital data grids of the square mesh. By putting

$$
h_{0}(z)=s H_{0}(z)
$$

a differential form of $H_{0}(z)$ with respect to $z$ is approximated as

$$
H^{\prime}(z)=\frac{h_{0}(z+s)-h_{0}(z-s)}{2 s^{2}} .
$$

Spatial differential forms of $u$ with respect to $x$ and $z$ may be taken similar to (12). On substituting these relations into (8), we obtain

$$
\begin{aligned}
u(x, z, t+\Delta t) & =u(x, z, t)+B[u(x+s, z, t)+u(x-s, z, t) \\
& +u(x, z+s, t)+u(x, z-s, t)-4 u(x, z, t) \\
& \left.-\frac{h_{0}(z+s)-h_{0}(z-s)}{2}\right]-C\left[\left\{\frac{u(x, z+s, t)-u(x, z-s, t)}{2}\right.\right. \\
& \left.\left.-h_{0}(z)\right\} \cos \pi x \sin \pi z-\frac{u(x+s, z, t)-u(x-s, z, t)}{2} \sin \pi x \cos \pi z\right],
\end{aligned}
$$

where $B$ and $C$ are dimensionless coefficients given by

$$
B=\frac{\Delta t}{4 \pi \sigma s^{2}}
$$

and

$$
C=\frac{\pi A \Delta t}{L s}
$$

$B$ being the coefficient of the terms which is related to diffusion of the magnetic field and $C$ the ones related to convection of the fluid, the ratio of the second to the first coefficient is correlated with the magnetic Reynolds' number by

$$
R=L C / B s=4 \pi^{2} \sigma A
$$


This parameter determines the behavior of the field. When $R$ is large, diffusion is dominated by convection. If we know the initial state $u(x, z, 0)$ and the spatial variation of $h_{0}(z)$ with respect to $z$, all the right-hand members of the equation (13) are known, and consequentely we get $u(x, z, \Delta t)$. As $u(x, z, \Delta t)$ is known, $u(x, z, 2 \Delta t)$ can next be calculated in a similar fashion. Repeating the same procedure, $u(x, z, 3 \Delta t), u(x, z, 4 \Delta t), \ldots \ldots$ can be successively obtained. $C$ is of course zero outside the convection cell.

\section{Models and Numerical Results}

\section{3-1. Uniform Magnetic Field}

In the first model, the author assumes that the magnetic field is uniform in the $x$ direction when the fluid is stationary, i.e. $H_{0}(z)=$ const. Weiss (1966) discussed magnetic fields associated with turbulent motions of the sun in a similar model.

In the model, (13) becomes

$$
\begin{aligned}
u(x, z, t+\Delta t) & =u(x, z, t)+B\{u(x+s, z, t) \\
& +u(x-s, z, t)+u(x, z+s, t)+u(x, z-s, t)-4 u(x, z, t)\}
\end{aligned}
$$

outside the cell. (17) is nothing but the equation of diffusion, the diffusivity being equal to $B$. It is important to note that, if we choose a large value of $B$, unstable solutions may be had because of errors accumulating in the successive procedures of computation. The solutions are sometimes oscillating. The condition for which the solutions of the equation of diffusion are stable is

$$
B \leqq 1 / 8
$$

in a two-dimensional case (Hagiwara, 1966). It is, in general, very difficult to estimate appropriate values of $B$ and $C$ which do not result in unstable solutions. According to the author's experience of trial and error, the solutions are sufficiently stable in the case that $B$ and $C$ are equal to 0.2 and 1.0 respectively. In this paper the author computes for the cases of $B=0.2$ and $C=0.2$, and $B=0.2$ and $C=1.0$. Weiss's models (1966) of convection cells are an isolated eddy and a double band of eddies, while the convection pattern the author computes in the present paper is a couple of eddies. An area occupied by one of the eddies being $L \times L$, the total area is $4 L$ ( $x$-direction) $\times 3 L$ ( $z$-direction). The grid spacing is determined as $s=L / 10$. Therefore, the total space concerned is covered with $41 \times 31$ grid points of square mesh, but the actual computation can be done with $21 \times 31$ grid points, considering a symmetry of the magnetic lines of force with respect to the $z$ axis passing through the boundary line between the two eddies. Letting $i$ and $j$ be the integer numbers, we can define $x=i s$ and $y=j s(i=1,2, \ldots \ldots, 21$ and $j=1,2, \ldots \ldots, 31)$. The boundary conditions are arbitrarily defined so that the magnetic field is identically constant at $j=1$ and $j=31$, and $\partial U / \partial x=$ const. and $\partial U / \partial z=$ const. at the margin of $i=1$. We see that $U(20 s, z, t)=U(22 s, z, t)$, considering the symmetry mentioned above.

It is natural that, the core of the actual earth having a radius of about $3500 \mathrm{~km}$, the scale of convection $L$ is estimated to be $3 \times 10^{3} \mathrm{~km}$. Assuming that $\sigma=3 \times 10^{-6}$ e.m.u., we get

$$
\Delta t=4 \pi \sigma s^{2} B=6.8 \times 10^{9} \mathrm{sec}(\fallingdotseq 210 \mathrm{yr} .)
$$


in the case of $B=0.2$, and $V$, a characteristic speed of the fluid, is

$$
V=\frac{\pi A}{L}=\frac{C s}{\Delta t} \fallingdotseq 8.8 \times 10^{-4} \mathrm{~cm} / \mathrm{sec}
$$

in the case of $B=0.2$ and $C=0.2$,i.e. $R=10$. When $R=50(B=0.2$ and $C=1.0)$, we have

$$
V \fallingdotseq 4.4 \times 10^{-3} \mathrm{~cm} / \mathrm{sec} \text {. }
$$

Rikitake (1967) estimated that the horizontal velocity of the convection at the surface of the core amounted to $10^{-3} \mathrm{~cm} / \mathrm{sec}$ or a little smaller at maximum. The former case $(R=10)$ may be rather true as compared with the latter case $(R=50)$ according to Rikitake's estimation. $V$ elocity streamlines (dotted line) and magnetic lines of force (solid line) of the initial field are shown in Fig. 1a, in which arrows show the directions of convecting current. Fig. $1 \mathrm{~b}$ shows the magnetic lines of force deformed slightly by the convection in the case of $R=10$ at the time $t=20 \Delta t \fallingdotseq 4200 \mathrm{yr}$.

The deformation has become large in Fig. 1c at $t=60 \mathrm{At}$. The final state is not reached till $t=200 \Delta t$, the figure of which is shown in Fig. 1d. The author also computes the lines of

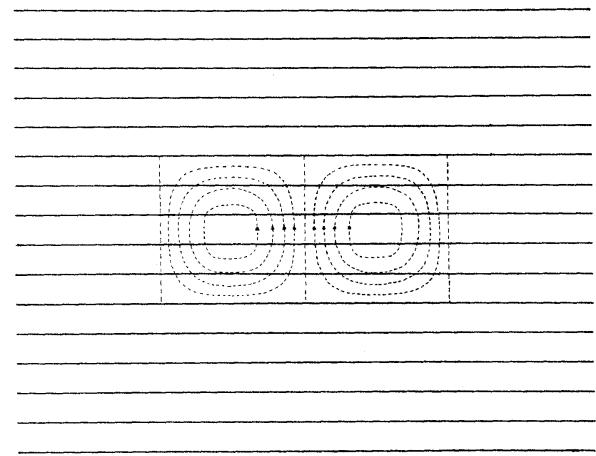

Fig. 1a Initial state of magnetic lines of force (solid line) and velocity streamlines (dotted line).

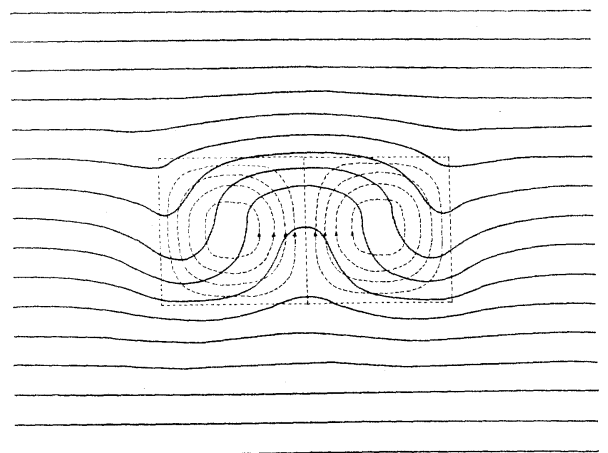

Fig. 1c Magnetic lines of force deformed by convection with $R=10$ at $t=60 \mathrm{At}$.

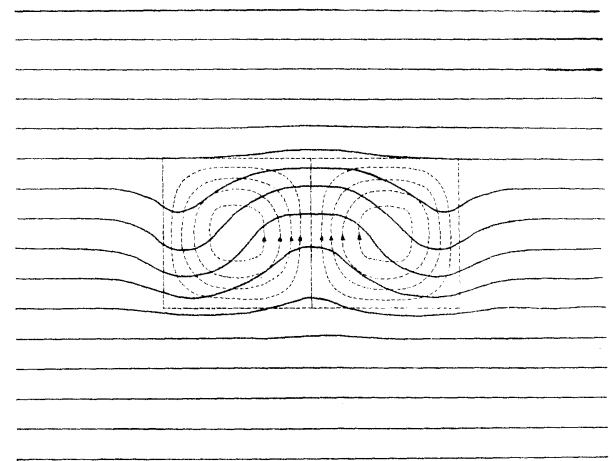

Fig. 1b Magnetic lines of force deformed by convection with $R=10$ at $t=20 \Delta t$.

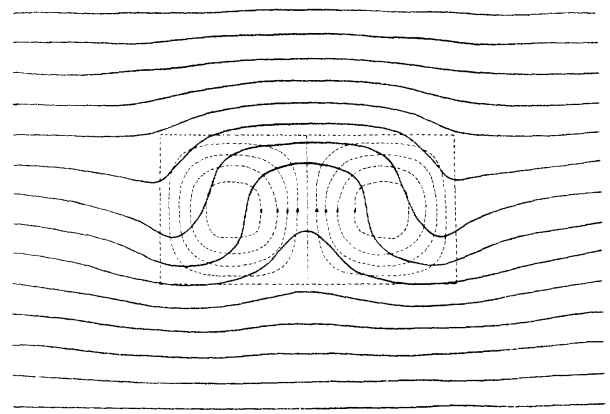

Fig. 1d Magnetic lines of force deformed by convection with $R=10$ at $t=200 \Delta t$, when the field approximately reaches a steady state. 


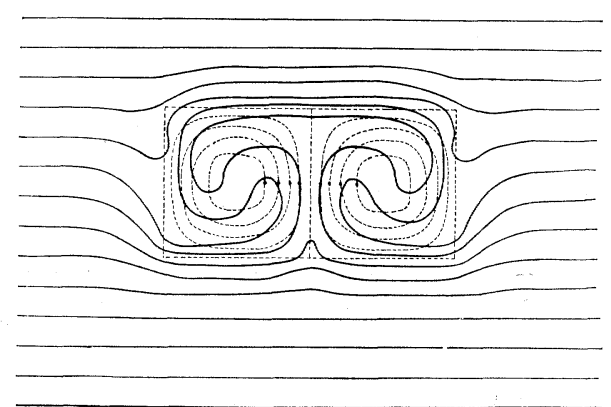

Fig. 2a Magnetic lines of force deformed by convection with $R=50$ at $t=20 \mathrm{At}$.

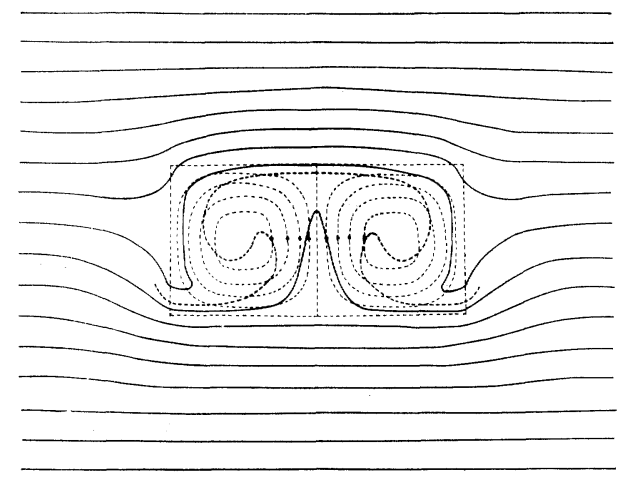

Fig. 2b Magnetic lines of force deformed by convection with $R=50$ at $t=60 \Delta t$.

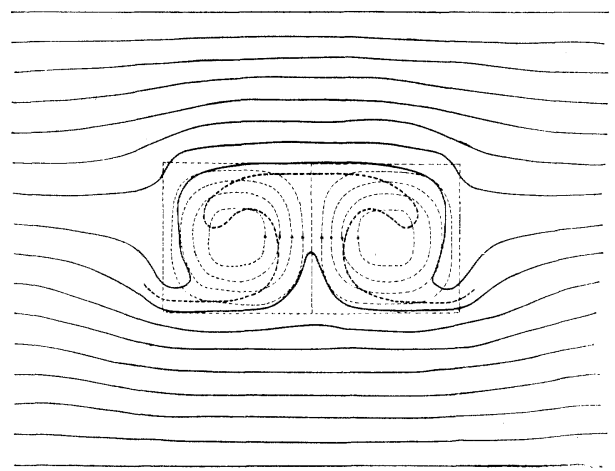

Fig. 2c Final state of magnetic lines of force associated with $R=50$ at $t=200 \mathrm{\Delta t}$.

force at the time $t=1000 \Delta t$, but the result is the same as that shown in Fig. 1d. This is evidence that Fig. 1d reaches the steady state.

When $R=50$, the computation is carried out in a similar fashion to the case of $R=10$. Figs. $2 \mathrm{a}, 2 \mathrm{~b}$ and $2 \mathrm{c}$ show the magnetic lines of force settled down by the times $t=20 \mathrm{At}$, $60 \Delta t$ and $200 \Delta t$, respectively. Although the deformation of the magnetic lines of force is remarkable, steady state is reached soon at about $t=200 \Delta t$.

The horizontal and the vertical components of the magnetic field are computed as

and

$$
H_{x}=-\{U(x, z+s, t)-U(x, z-s, t)\} / 2 s
$$

$$
H_{z}=\{U(x+s, z, t)-U(x-s, z, t)\} / 2 s
$$

in the digital computation respectively, $z$ being positive downwards. Figs. 3a and 3b show, respectively, the horizontal and the vertical components of the field corresponding to the time $t=200 \Delta t(R=10)$. When $R=50$ and $t=200 \Delta t$ the magnetic patterns become extremely complicated as shown in Figs. $3 \mathrm{c}$ and $3 \mathrm{~d}\left(H_{x}\right.$ add $H_{z}$, respectively). The contour values are given in units of $H_{0}(z)=$ const. In order to see the spatial variation of the non-dipole field caused by a convective up-flow, the author calculates the magnetic field on the line $j=6 s$, for example. 


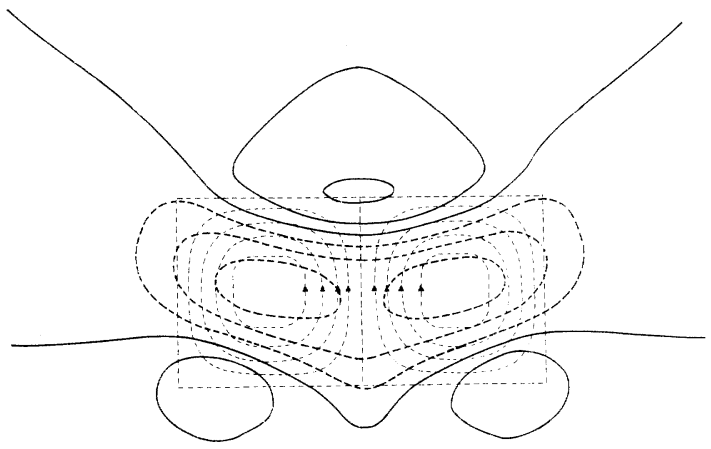

Fig. 3a Horizontal component of magnetic field calculated from Fig. 1d.

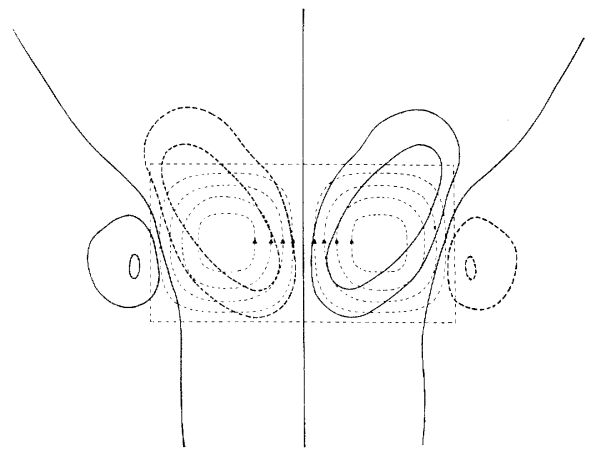

Fig. 3b Vertical component of the magnetic field calculated from Fig. 1d.

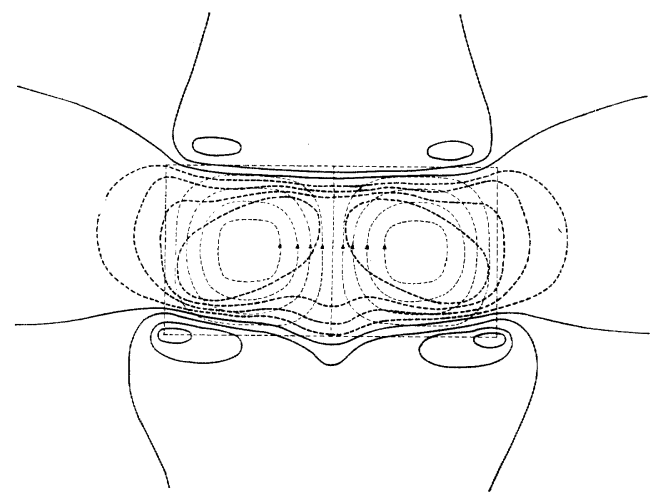

Fig. 3c Horizontal component of the magnetic field calculated from Fig. 2c.

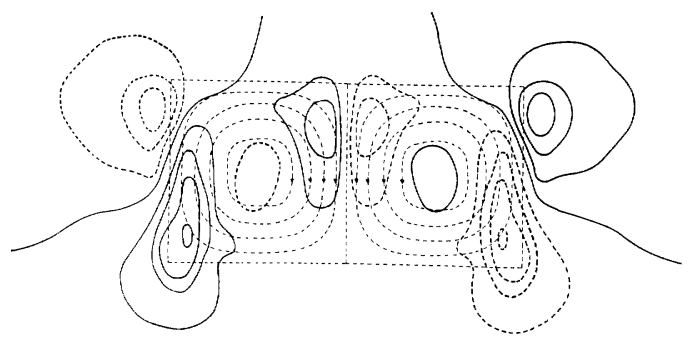

Fig. 3d Vertical component of the magnetic field calculated from Fig. 2c.

Figs. $4 \mathrm{a}$ and $4 \mathrm{~b}$ show the distributions of the horizontal and the vertical components of the field along the line $j=6 s$, which are obtained from Figs. $3 \mathrm{a}$ and $3 \mathrm{~b}$, respectively. A horizontal high and a vertical zero are seen just above the up-flow. The results estimated from these figures are qualitatively coincident with the non-dipole field actually observed over Africa. The magnetic distributions over a down-flow, which are obtained from Figs. 3a and $3 \mathrm{~b}$ are shown in Figs. $4 \mathrm{c}$ and $4 \mathrm{~d}$ respectively. A horizontal low is seen above the down-flow. Although it is difficult to get detailed quantitative results from Figs. 4c and $4 \mathrm{~d}$, it may possibly be proved that the field weakening over the Pacific is associated with a down-flow in the core's top layer.

\section{3-2. Non-Uniform Magnetic Field}

According to Bullard and Gellman's dynamo (1954), the intensity of the toroidal field is radially non-uniform and a maximum of field intensity occurs approximately at a point some $2 / 3$ radius distant from the center of the core. Such a toroidal field may be approximated by an initial magnetic field such as

$$
H_{0}(z)=\sin ^{2} \frac{\pi(L-z)}{L} \quad \text { for } L \leqq z \leqq 2 L .
$$

The strongest magnetic field intensity is found on the line passing through the two centers 


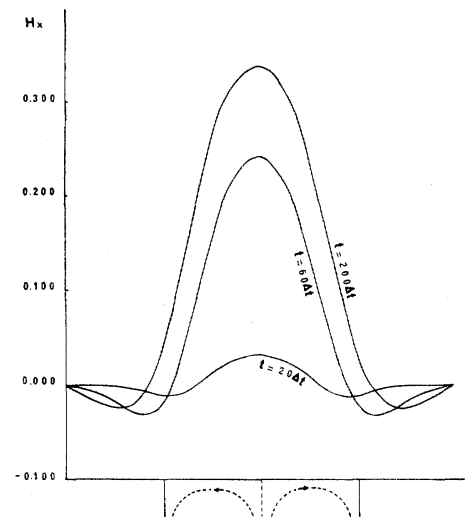

Fig. 4a Horizontal component distribution over a convective up-flow.

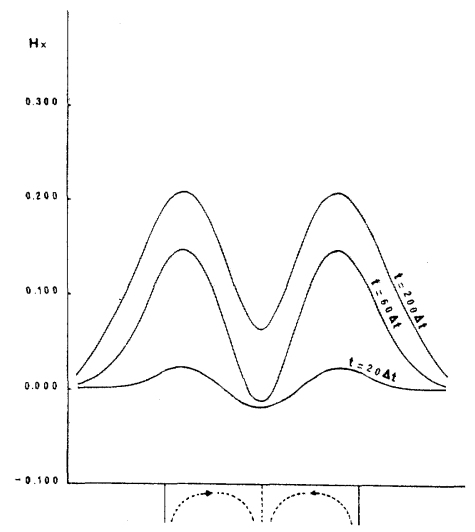

Fig. 4c Horizontal component distribution over a convective down-flow.

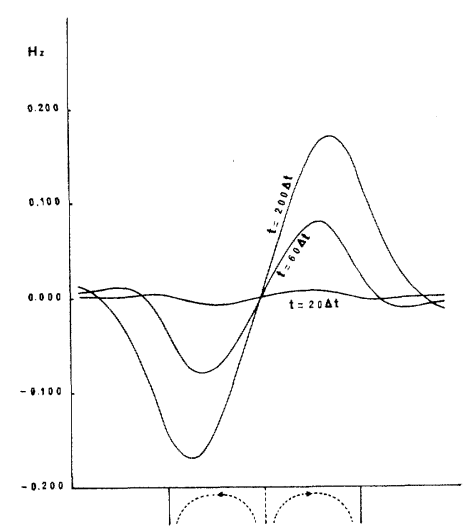

Fig. 4b Vertical component distribution over a convec. tive up-flow.

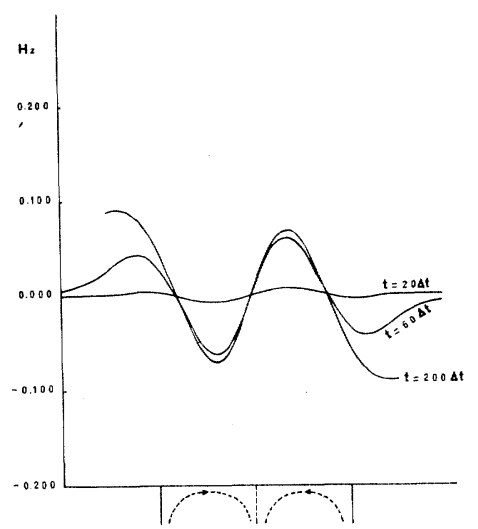

Fig. 4d Vertical component distribution over a convective down-flow.

of the convection cells. However, it should be borne in mind in this case that the equation of diffusion is different from (17) outside the convective cell because $h_{0}(z+s)=h_{0}(z-s)$ in (13), i.e. the initial magnetic field given by (20) is diffusive even without any convecting currents. Therefore, we should pay attention to the fact that the model whose initial magnetic field is given by (20) is appropriate for a model of the toroidal field only before the diffusion has not become considerable. Although computations for a large value of $t$ after the diffusion effect predominates have no meaning, it is interesting to observe the characteristic phenomena displayed with this model. Fig. 5 a shows the initial magnetic lines of force and the velocity streamlines. Figs. $5 b, 5 c$ and $5 d$ show the lines of force distorted gradually by convection currents in the case $R=10$ at $t=20 \Delta t, 60 \Delta t$ and $200 \Delta t$ respectively. The behavior of the magnetic field in the case of $R=50$ is also computed, as appears in Figs. 6a, 6b, and $6 \mathrm{c}$, which are corresponding to the times of $20 \Delta t, 60 \Delta t$ and $200 \Delta t$, respectively. The magnetic fields almost reach the final steady state in both the cases of $R=10$ and $R=50$ at $t=$ $200 \Delta t$. 

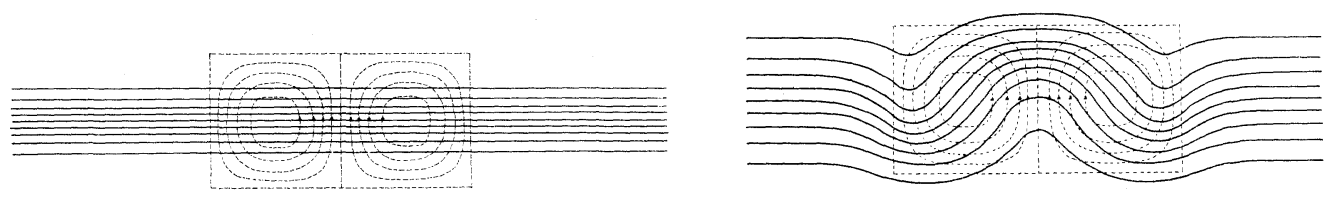

Fig. 5a Initial state of magnetic lines of force and velocity streamlines (non-uniform magnetic field).

Fig. 5b Magnetic lines of force deformed by convection with $R=10$ at $t=20 \Delta t$ (non-uniform magnetic field).

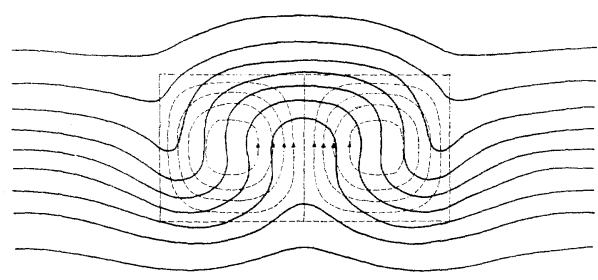

Fig. 5c Magnetic lines of force deformed by convection with $R=10$ at $t=60 \Delta t$ (nonuniform magnetic field).
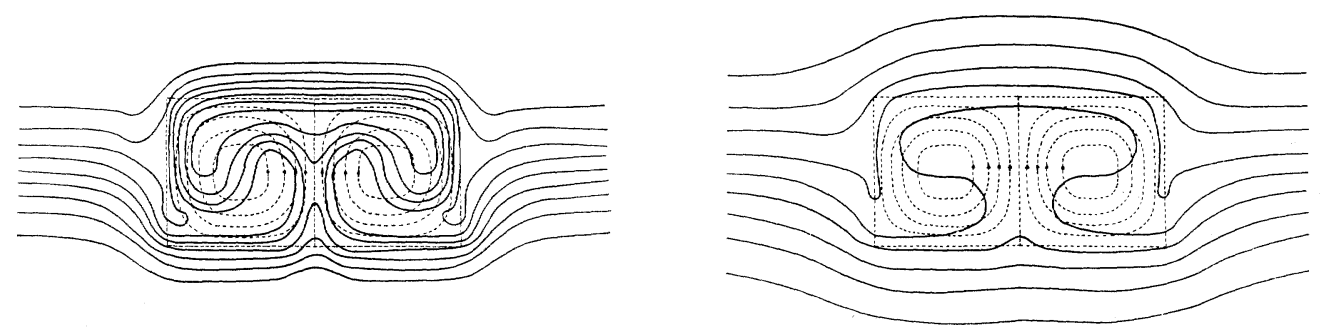

Fig. 6a Magnetic lines of force deformed by convection with $R=50$ at $t=20 \Delta t$ (nonuniform magnetic field).

Fig. 6b Magnetic lines of force deformed by convection with $R=50$ at $t=60 \mathrm{At}$ (non-uniform magnetic field).

Fig. 5d Magnetic lines of force deformed by convection with $R=10$ at $t=200 \Delta t$ (non-uniform magnetic field). 


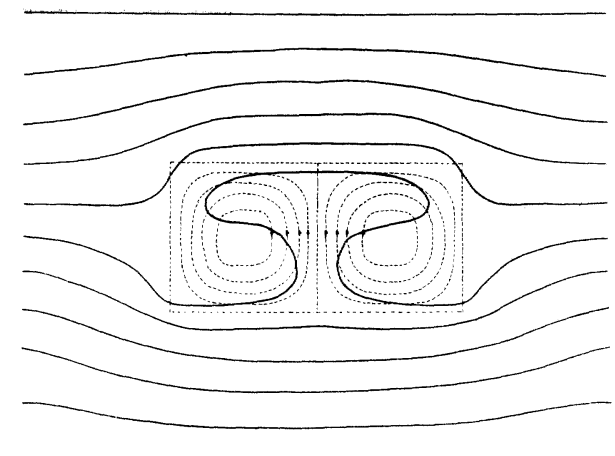

Fig. 6c Magnetic lines of force deformed by convection with $R=50$ at $t=200 \Delta t$ (non-uniform magnetic field).

\section{Concluding Remarks}

The results obtained in this paper indicate that an up-flow and a down-flow of convection within the earth's core may possibly induce the non-dipole high and low at the earth's surface. What are shown in the figures are the time-dependent magnetic lines of force deformed by convecting currents of a conducting fluid. The distributions of the horizontal and the vertical components on the earth's surface are also roughly estimated. Although no detailed discussion is possible from the results mentioned above, a qualitative correlation between the magnetic field and convection of the core material is found.

The mathematical method used here is thought to be one of the best methods to com. pute non-steady problems, the equation of which is expressed as a "parabolic type" with respect to time as in an equation of diffusion. The fluid motion is defined as given by formula (7) in this paper. Deformation of magnetic lines of force will be studied by means of this method in a later paper for a more general case in which the electromagnetic force exerted to the fluid is taken into account.

The author is grateful to Prof. T. Rikitake from whose suggestions this work was undertaken.

\section{References}

Bullard, E.C., Freedman, C., Gellman, H. and Nixon, J., Phil. Trans. Roy. Soc. London A, 243, 67, 1950. Bullard, E.C. and Gellman, H., Phil. Trans. Roy. Soc. London A, 247, 213, 1954.

Hagiwara, Y., Bull. Earthq. Res. Inst., 44, 1537, 1966.

Nagata, T. and Rikitake, T., J. Geomag. Geoelec., 13, 42, 1961.

Rikitake, T., J. Geomag. Geoelec., 19, 1967.

Vestine, E.H., J. Geomag. Geoelec., 17, 165, 1965.

Weiss., N.O., Proc. Roy. Soc., Ser. A, 293, 310, 1966. 